Article

\title{
The Protective Effect of Rhizoma Dioscoreae Extract against Alveolar Bone Loss in Ovariectomized Rats via Regulating Wnt and p38 MAPK Signaling
}

\section{Zhiguo Zhang ${ }^{1}$, Lihua Xiang ${ }^{1}$, Dong Bai ${ }^{1}$, Wenlai Wang ${ }^{1}$, Yan Li ${ }^{1}$, Jinghua Pan ${ }^{1}$, Hong Liu ${ }^{1}$, Shaojun Wang ${ }^{1}$, Gary Guishan Xiao ${ }^{2,3, *}$ and Dahong Ju ${ }^{1, *}$}

1 Institute of Basic Theory, China Academy of Chinese Medical Sciences, Beijing 100700, China; E-Mails: zzgtcm@163.com (Z.Z.); xlh891201@sina.com (L.X.); abai2000_0@163.com (D.B.); wangwenlai666@163.com (W.W.); lei_ruo@163.com (Y.L.); jh-p@163.com (J.P.); liuhong@163.com (H.L.); wangshaojun@163.com (S.W.)

2 School of Pharmaceutical Science, Dalian University of Technology, Dalian 116024, China

3 Functional Genomics and Proteomics Laboratory, Osteoporosis Research Center, Creighton University Medical Center, Omaha, NE 68131, USA

* Authors to whom correspondence should be addressed; E-Mails: gxiao@creighton.edu (G.G.X.); judahong@sohu.com (D.J.); Tel.: +86-411-84986473 or +1-402-280-5911 (G.G.X.); +86-10-84024005 (D.J.); Fax: +1-402-280-4284 (G.G.X.); +86-10-64013896 (D.J.).

Received: 22 September 2014; in revised form: 28 November 2014 / Accepted: 2 December 2014 / Published: 12 December 2014

Abstract: Aim: The aim of this study was to evaluate the osteoprotective effect of aqueous Rhizoma Dioscoreae extract (RDE) on the alveolar bone of rats with ovariectomy-induced bone loss. Methods: Female Wistar rats were subjected to either ovariectomy or a sham operation (SHAM). The ovariectomized (OVX) rats were treated with vehicle (OVX) or RDE by oral gavage or with $17 \beta$-estradiol (E2) subcutaneously. After treatments, the bone mineral density (BMD), the three-dimensional bone architecture of the alveolar bone and the plasma biomarkers of bone turnover were analyzed to assess bone metabolism, and the histomorphometry of the alveolar bone was observed. Microarrays were used to evaluate gene expression profiles in alveolar bone from RDE-treated and OVX rats. The differential expression of genes was further analyzed using Ingenuity Pathway Analysis (IPA). The key findings were verified using real-time quantitative RT-PCR (qRT-PCR). Results: Our results showed that RDE inhibited alveolar bone loss in OVX rats. Compared to the OVX rats, the RDE-treated rats showed upregulated expression levels of 207 genes and downregulated expression levels of 176 genes in the alveolar bone. The IPA showed that several genes had 
the potential to code for proteins that were involved in the $\mathrm{Wnt} / \beta$-catenin signaling pathway (Wnt7a, Fzd2, Tcf3, Spp1, Frzb, Sfrp2 and Sfrp4) and the p38 MAPK signaling pathway (Il1rn and Mapk14). Conclusion: These experiments revealed that RDE could inhibit ovariectomy-induced alveolar bone loss in rats. The mechanism of this anti-osteopenic effect in alveolar bone may be involved in the reduced abnormal bone remodeling, which is associated with the modulation of the Wnt/ $\beta$-catenin and the p38 MAPK signaling pathways via gene regulation.

Keywords: alveolar bone loss; gene expression profile; herbal medicine; ovariectomized rats; Rhizoma Dioscoreae

\section{Introduction}

Bone remodeling is a lifelong dynamic process in which mature bone tissue is removed from the skeleton and new bone tissue is formed. Abnormal bone metabolism is due to imbalanced bone formation and resorption, which often occurs in estrogen-deficient ovariectomized (OVX) rats and postmenopausal women [1,2]. Alveolar bone is essential for the support of teeth, which are anchored to the bone by desmodontal fibers. Previous research has shown that osteoporosis may reduce alveolar bone mass and alter alveolar bone structure [3]. This bone loss occurs rapidly during the early postmenopausal period and levels off approximately six years after menopause, likely due to the decrease in estrogen production in postmenopausal women [4]. There exists a relationship between alveolar bone loss and osteoporosis in postmenopausal women [5]. The study showed that alveolar bone loss was significantly correlated with the bone mineral density (BMD) of the trochanter, Ward's triangle and femur of postmenopausal women [6]. Postmenopausal women with osteoporosis have a greater chance of having alveolar bone loss than do those without osteoporosis [7]. Alveolar bone loss leads to mobile teeth or tooth loss, which seriously reduce the quality of life of postmenopausal women [8].

Estrogen [9], bisphosphonates [10] or parathyroid hormone (PTH) [11] have been used to prevent postmenopausal alveolar bone loss, but many lines of evidence indicate that long-term treatments with those drugs might cause adverse reactions, such as an increased risk of ovarian and endometrial cancer [12,13], osteonecrosis of the jaws [14], nervous system disorders [15] and venous thromboembolism [16]. Thus, an alternative therapeutic strategy with proven efficacy and safety should be developed to prevent and treat alveolar bone loss. For years, Chinese herbal medicine has been widely used in clinical practice to treat bone diseases and will most likely continue to be used as a cost-effective alternative medicine in China [17]. Rhizoma Dioscoreae (RD) is the dried rhizome of Dioscorea opposite Thunb. and has been used to strengthen bone for a long time in China. Our previous studies indicated that treatment with Rhizoma Dioscoreae extract (RDE) protects against bone loss of the peripheral skeleton in ovariectomized (OVX) rats [18], a model of postmenopausal osteoporosis. This led us to question whether RDE had a similar protective effect in alveolar bone loss, though the alveolar bone was morphologically and functionally different from other bones of the axial or peripheral skeleton. The aims of this study were to analyze the anti-osteopenic effect of RDE on alveolar bone in OVX rats and to explore the molecular targets of RDE. 


\section{Experimental Section}

\subsection{Preparation of Aqueous Extract}

The preparation of RDE was performed as we previously reported [18]. In this study, we used the same batch of RDE as presented previously.

\subsection{Animal Grouping and Treatments}

Many studies have used 6-month-old female rats that have undergone a bilateral ovariectomy to model postmenopausal osteoporosis [19,20]. We obtained a total of forty-eight 6-month-old virgin Wistar rats with a body weight of $310 \pm 20.0$ g from the Experimental Animal Center of the Academy of Military Medical Sciences (SCXK-(Military) 2002-001, Beijing, China). The Institutional Ethics Committee of the China Academy of Chinese Medical Sciences approved the experimental research on the animals (Approval Number 2012-006). The acclimatized rats were either sham-operated (SHAM, $n=12$ ) or bilaterally OVX $(n=36)$ using the dorsal approach [21]. The OVX rats were randomly divided into three groups: OVX group (OVX, $n=12$ ); 17 $\beta$-estradiol treatment group (E2, $n=12$ ); RDE group (RDE, $n=12$ ). 17 $\beta$-Estradiol (Sigma-Aldrich, Saint Louis, MO, USA) was prepared by dissolving a small amount in ethanol and adjusting the volume using olive oil. The rats in the E2 group were treated with daily subcutaneously administered $17 \beta$-estradiol ( $30 \mu \mathrm{g} / \mathrm{kg}$ body weight). The rats in the RDE group were treated with RDE dissolved in distilled water at $1.3 \mathrm{~g} / \mathrm{kg}$ body weight/day by oral gavage. The gavage dosage was based on the recommended dosage for humans (30 g/day) according to the Chinese Pharmacopeia, adjusted for the rat/human body mass ratio. The rats in the SHAM and the OVX groups were administered the same volume of distilled water by oral gavage. All rats were fed standard chow during the course of the experiments (Animal Center of the Fourth Military Medical University, Xi'an, China). All treatments started 1 week after surgery and continued for 12 weeks.

\subsection{Preparation of Specimens}

The day after the last treatment, the animals were anesthetized with an intraperitoneal injection of ketamine ( $80 \mathrm{mg} / \mathrm{kg}$ body weight) and xylazine (12 mg/kg body weight) and sacrificed by exsanguination. We obtained blood samples by puncturing the abdominal aorta before death; we collected the blood samples in heparinized tubes. The blood samples were centrifuged at $3000 \times g$ at $4{ }^{\circ} \mathrm{C}$ for $10 \mathrm{~min}$, aliquoted and frozen at $-80^{\circ} \mathrm{C}$ until the samples were used for assay. The right mandibles were dissected, filled with physiological saline and stored at $-20{ }^{\circ} \mathrm{C}$ for measurements of BMD and microstructure by micro-computerized tomography (micro-CT). After the measurement of micro-CT, the right mandibles were used for histological observation. The left mandibles were dissected and stored at $-80{ }^{\circ} \mathrm{C}$ for microarray and real-time quantitative RT-PCR (qRT-PCR) assays.

\subsection{Biochemical Markers of Bone Turnover}

The plasma levels of bone formation marker, procollagen type $1 N$-terminal propeptide (P1NP), and bone resorption marker, $C$-terminal cross-linked telopeptides of type I collagen (CTX), were assessed using the enzyme-linked immunosorbent assay (Immunodiagnostic Systems Ltd., Boldon, UK) for 
control, standard and duplicate tests. Absorbance was read using an ELISA reader (Bio-Tek, Colmar, France) at $450 \mathrm{~nm}$.

\subsection{Micro-CT Analysis}

The right mandible of each animal, without sample preparation or decalcification, was scanned with a high-resolution micro-CT (SkyScan 1172 micro-CT system, Antwerp, Belgium). The SkyScan micro-CT uses the cone-beam reconstruction method to determine the conical geometry of the X-ray source. The desktop SkyScan micro-CT system was used according to a method previously described [22]. Each sample was placed on the rotational stage and translated along the continuously variable magnification stage to achieve the desired resolution $(6.8 \mu \mathrm{m})$. The sample was rotated $185^{\circ}$, and the images were obtained every $0.9^{\circ}$. Repeated scans were performed at the beginning of the experiment to verify the reproducibility of the method. A low-pass filter was used to remove noise from the resulting gray-scale images. The trabecular bone was determined by a fixed threshold.

After the images were captured $(100 \mathrm{keV}, 100 \mu \mathrm{A})$, we used CTanalyser, an image analysis software of SkyScan, to establish a square region of interest (ROI) $(1 \mathrm{~mm} \times 1 \mathrm{~mm})$ on sagittal images of the first molar. All selected ROIs had no overlapping areas with tooth roots. A cubic region $(1 \mathrm{~mm} \times 1 \mathrm{~mm} \times 1 \mathrm{~mm})$ beginning $1.5 \mathrm{~mm}$ beneath the lowest point of the first molar crown was established as the "volume of interest” (VOI) using NRecon, a three-dimensional 3D reconstruction software from SkyScan. We performed morphological measurements of the trabecular bone within the VOI using the standard SkyScan software package. We used three-dimensional analyses to assess the BMD, bone volume fraction (BV/TV), the trabecular thickness (Tb.Th), the trabecular separation (Tb.Sp), the trabecular number (Tb.N), the structural model index (SMI) and the degree of anisotropy (DA) for the same VOI [23].

\subsection{Histological Observation}

The right mandibles were fixed in 10\% buffered formalin, decalcified in 14\% EDTA, dehydrated and embedded in paraffin. Sections were cut using a standard microtome (Leica DMB6000B and CTR6000, Leica, Wetzlar, Germany), affixed to glass slides and stained with hematoxylin and eosin.

\subsection{Microarray Data Analysis}

Alveolar bone was prepared from six RDE rats and six OVX rats. The gene microarray assay was conducted by KangChen Bio-tech (Shanghai, China). We performed a microarray analysis of whole-genome gene expression profiling using Agilent chips $(4 \times 44 \mathrm{~K}$, Agilent Technologies), which consisted of approximately 41,000 probes for rat genes. The total RNA of distal right alveolar bone from the RDE group and OVX group was harvested using TRIzol (Invitrogen, Carlsbad, CA, USA) and the RNeasy kit (Qiagen, Chatsworth, CA, USA), according to the manufacturer's instructions, including a DNase digestion step. After RNA measurement using the NanoDrop ND-1000 (NanoDrop Technologies, Montchanin, DE, USA)and denaturing gel electrophoresis, the samples were amplified and labeled using an Agilent Quick Amp labeling kit and hybridized with an Agilent whole genome oligo microarray in Agilent's Sure-Hyb Hybridization Chambers (Agilent Technologies, Santa Clara, CA, USA). After hybridization and washing, the processed slides were scanned with the Agilent DNA 
microarray scanner using settings recommended by Agilent Technologies. The resulting text files extracted from Agilent Feature Extraction Software were imported into Agilent GeneSpring software (Agilent, version 11.0, Agilent Technologies, Santa Clara, CA, USA) for further analysis. The gene expression was normalized to the OVX group. The differentially expressed genes were identified through the fold change and $t$-test $p$-value screening.

\subsection{Ingenuity Pathway Analysis}

To facilitate the gene microarray data analysis and to relate specific genes to the underlying biological processes, Ingenuity Pathway Analysis (IPA) (Ingenuity ${ }^{\circledR}$ Systems, Redwood, CA, USA) was used for the function and pathway analysis. Differentially expressed genes between the RDE group and the OVX group were imported into IPA, and the top five canonical pathways were observed. The canonical pathway analysis identified the molecular pathways from the IPA library of canonical pathways that were most significant to the dataset. The genes from the dataset that were associated with a canonical pathway were considered for additional analysis.

\subsection{Quantitative Real-Time RT-PCR ( $q R T-P C R)$}

The total RNA was purified using an RNeasy Mini Kit (Qiagen, Valencia, CA, USA), and $4 \mu \mathrm{g}$ RNA was reverse-transcribed using the Superscript First Strand synthesis system (Invitrogen, Carlsbad, CA, USA) to cDNA. The qRT-PCR amplification was performed using the SYBR-green detection of PCR products in real time with an ABI-7500 Sequence Detection System (Applied Biosystems, Foster City, CA, USA) according to the manufacturer's instruction. The primers used in the qRT-PCR analysis are presented in Table 1 . The PCR program was initiated by $10 \mathrm{~s}$ at $95^{\circ} \mathrm{C}$ before 40 thermal cycles, each of $5 \mathrm{~s}$ at $95^{\circ} \mathrm{C}$ and $34 \mathrm{~s}$ at $60{ }^{\circ} \mathrm{C}$. We analyzed the data according to the $2^{-\Delta \Delta \mathrm{Ct}}$ method and normalized to the glyceraldehyde-3-phosphate dehydrogenase (GAPDH) expression in each sample. The melting curves for each PCR reaction were generated to ensure the purity of the amplification product. A no-template negative control was included in each experiment.

Table 1. Primers used for qRT-PCR analysis.

\begin{tabular}{cl}
\hline Transcript & Sequence (5'-3') \\
\hline \multirow{2}{*}{ Gapdh } & F: 5'-GGGAAACTGTGGCGTGAT-3' \\
& R: 5'-GAGTGGGTGTCGCTGTTGA-3' \\
\hline \multirow{2}{*}{ Fzd2 } & F: 5'-CAGGGCACTAAGAAAGAAGGCT-3' \\
& R: 5'-AGGAACCAGGTGAGGGACAGA-3' \\
\hline \multirow{2}{*}{ Il1rn } & F: 5'-CTTACCTTCATCCGCTCCGA-3' \\
& R: 5'-GATCAGGCAGTTGGTGGTCAT-3' \\
\hline \multirow{2}{*}{ Mapk14 } & F: 5'-ATAGACGAATGGAAGAGCCTGAC-3' \\
& R: 5'-CAAAGATACATGGACAAACGGAC-3' \\
\hline \multirow{2}{*}{ Sfrp2 } & F: 5'-CCGAAAGGGACCTGAAGAAAT-3' \\
& R: 5'-ACCAGATACGGAGCGTTGATG-3' \\
\hline \multirow{2}{*}{ Sfrp4 } & F: 5'-AAGTCTTTGTCACCTATCCCTCG-3' \\
& R: 5'-CGGCTGGCTATCTGCTTCTT-3' \\
\hline
\end{tabular}


Table 1. Cont.

\begin{tabular}{cl}
\hline \multirow{2}{*}{ Spp1 } & F: 5'-TTTCACTCCAATCGTCCCTACA-3' \\
& R: 5'-AGTCCATAAGCCAAGCTATCACC-3' \\
\hline \multirow{2}{*}{$T c f 3$} & F: 5'-ACAGTCTCAGCAGCAAATCCAA-3' \\
& R: 5'-GAAGACGCAGGGCTATCACAA-3' \\
\hline \multirow{2}{*}{ Ptk2b } & F: 5'-TCTGTGACCCGTCTACCCATC-3' \\
& R: 5'-CTTTCTCCAGCACTCCGATGA-3' \\
\hline \multirow{2}{*}{$W n t 7 a$} & F: 5'-CTCTGCCGACATCCGCTAC-3' \\
& R: 5'-CGACCCGCCTCGTTATTG-3' \\
\hline \multirow{2}{*}{ Bmp1 } & F: 5'-CAAAGGACCCGACTCAGCA-3' \\
& R: 5'-CCACATAGTCATACCAGCACAGG-3' \\
\hline \multirow{2}{*}{ Csf1r } & F: 5'-AAGCCGAAATATCAGGTGCG-3' \\
& R: 5'-GGGTCGATGAAGGTGTAGTTGTT-3' \\
\hline \multirow{2}{*}{$F r z b$} & F: 5'-TCCAAGGGATACCGTCAACC-3' \\
& R: 5'-ATCCTTCCACTTCTCAGCGATAG-3' \\
\hline
\end{tabular}

\subsection{Western Analysis}

Alveolar bone was crushed in liquid nitrogen to extract bone proteins and solubilized in radioimmunoprecipitation assay (RIPA) buffer with protease inhibitors and phosphatase inhibitors. Insoluble substances were separated and removed by centrifugation at $10,000 \mathrm{rpm}$ for $5 \mathrm{~min}$ at $4{ }^{\circ} \mathrm{C}$. A commercial BCA reagent (Pierce, Rockford, IL, USA) was used to determine protein concentrations. Eighty micrograms of proteins from each sample were resolved, used for electrophoresis on $12 \%$ SDS-polyacrylamide gels and transferred onto nitrocellulose membranes (Hybond-ECL; GE Healthcare, Piscataway, NJ, USA). Membranes were blocked with 5\% nonfat dry milk for $1 \mathrm{~h}$, incubated overnight at $4{ }^{\circ} \mathrm{C}$ with antibodies against Tcf-3 (1:1000 dilution) (Santa Cruz Biotechnology, Santa Cruz, CA, USA), p38 $\alpha$ (1:1000 dilution) (Santa Cruz Biotechnology) and $\beta$-actin (1:25,000 dilution) (SigmaAldrich, St. Louis, MO, USA). Subsequently, membranes were washed and incubated for $1 \mathrm{~h}$ using an HRP-linked antibody (1:1000 dilution, Cell signaling Technology, Beverly, MA, USA). For the detection of immunoreactive proteins, an enhanced chemiluminescence kit (PerkinElmer, Waltham, MA, USA) was used. Using Quantity One software (Bio-Rad, Hercules, CA, USA), the intensities of specific bands were quantified with densitometry and then were normalized for $\beta$-actin. Normalized data are expressed as the fold increase vs. the SHAM or OVX control.

\subsection{Statistical Analysis}

All values were expressed as the mean \pm standard deviation. All analyses were conducted using the SPSS 13.0 (SPSS Inc., Chicago, IL, USA). The difference between the groups regarding the evaluated parameters was tested using the analysis of variance (ANOVA) followed by the least significant difference (LSD) test. The data of all groups passed the Kolmogorov-Smirnov test of normality. $p<0.05$ was considered to be statistically significant. 


\section{Results}

\subsection{Effect of RDE on Biomarkers of Bone Turnover}

The plasma levels of bone formation (procollagen type $1 \mathrm{~N}$-terminal propeptide, P1NP) and bone resorption (CTX) biomarkers after a 12-week treatment of different rat groups are provided in Figures 1 and 2. At the end of Week 12, the OVX group showed a significantly higher concentration of P1NP or CTX than the SHAM group $(p<0.01)$. Moreover, the E2 and RDE group showed a significantly lower $(p<0.01$ or $p<0.05)$ level of P1NP or CTX than the OVX group.

Figure 1. Effect of Rhizoma Dioscoreae extract (RDE) on (procollagen type $1 \mathrm{~N}$-terminal propeptide) P1NP in plasma after 12 weeks of treatment. ${ }^{a} p<0.01$ vs. the SHAM group; ${ }^{\mathrm{b}} p<0.05$ vs. the ovariectomized (OVX) group; ${ }^{\mathrm{c}} p<0.01$ vs. the OVX group. E2, 17 $\beta$-estradiol.

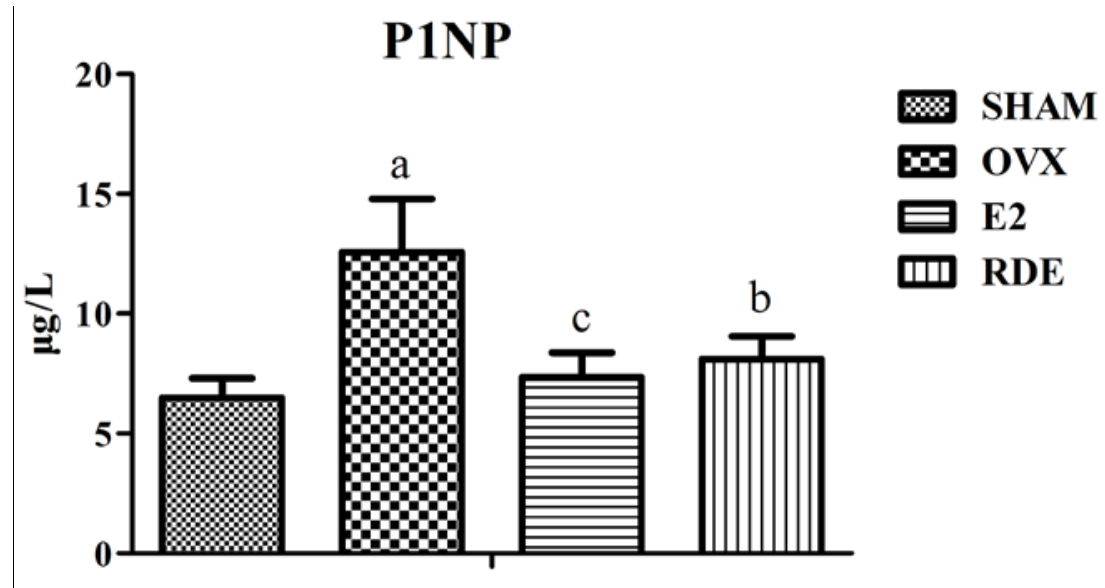

Figure 2. Effect of RDE on C-terminal cross-linked telopeptides of type I collagen (CTX) in plasma after 12 weeks of treatment. ${ }^{\mathrm{a}} p<0.01$ vs. the SHAM group; ${ }^{\mathrm{b}} p<0.01 \mathrm{vs}$. the OVX group.

\section{CTX}

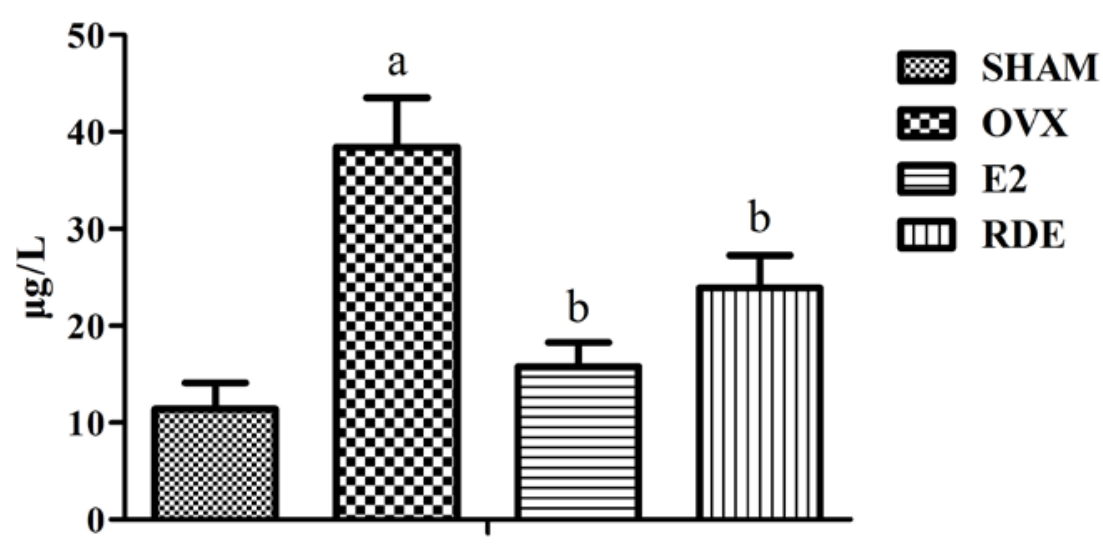

\subsection{Effect of RDE on BMD and Trabecular Bone Microarchitecture}

We evaluated the BMD and trabecular bone microarchitecture of four groups. Analysis of the alveolar bone morphometric parameters indicated that ovariectomy significantly decreased the BMD, trabecular 
BV/TV, Tb.N and Tb.Th $(p<0.01)$ and increased Tb.Sp, SMI and DA $(p<0.01)$ compared to the SHAM group. Treatment with RDE or E2 significantly inhibited the OVX-induced changes (Table 2). Further, treatment with E2 or RDE relieved damage to the trabecula in alveolar bone induced by the ovariectomy (Figure 3a-d).

Table 2. Effect of RDE on bone mineral density (BMD) and trabecular bone microarchitecture. BV/TV, bone volume fraction; Tb.Th, trabecular thickness; Tb.Sp, trabecular separation; Tb.N, trabecular number; SMI, structural model index; DA, degree of anisotropy.

\begin{tabular}{ccccc}
\hline Parameters & SHAM & OVX & E2 & RDE \\
\hline BMD $\left(\mathrm{g} / \mathrm{cm}^{3}\right)$ & $0.348 \pm 0.005$ & $0.295 \pm 0.006^{\mathrm{a}}$ & $0.328 \pm 0.008^{\mathrm{c}}$ & $0.313 \pm 0.004^{\mathrm{b}}$ \\
$\mathrm{BV} / \mathrm{TV}(\%)$ & $55.100 \pm 4.718$ & $6.777 \pm 4.1569^{\mathrm{a}}$ & $42.756 \pm 0.843^{\mathrm{c}}$ & $22.299 \pm 2.535^{\mathrm{c}}$ \\
Tb.Th $(\mu \mathrm{m})$ & $81.592 \pm 8.654$ & $41.237 \pm 2.592^{\mathrm{a}}$ & $74.226 \pm 6.361^{\mathrm{c}}$ & $49.382 \pm 3.976^{\mathrm{c}}$ \\
Tb.Sp $(\mu \mathrm{m})$ & $181.62 \pm 26.48$ & $678.27 \pm 33.21^{\mathrm{a}}$ & $319.63 \pm 95.91^{\mathrm{c}}$ & $519.29 \pm 13.55^{\mathrm{c}}$ \\
Tb.N $(1 / \mathrm{mm})$ & $6.84 \pm 1.28$ & $1.61 \pm 0.91^{\mathrm{a}}$ & $5.81 \pm 0.61^{\mathrm{c}}$ & $4.52 \pm 0.40^{\mathrm{c}}$ \\
SMI & $0.914 \pm 0.275$ & $2.327 \pm 0.419^{\mathrm{a}}$ & $1.637 \pm 0.217^{\mathrm{c}}$ & $1.712 \pm 0.379^{\mathrm{c}}$ \\
DA & $2.526 \pm 0.837$ & $7.770 \pm 0.679^{\mathrm{a}}$ & $2.185 \pm 0.271$ & $1.861 \pm 0.188$ \\
\hline
\end{tabular}

Values are presented as the means $\pm \mathrm{SD}\left(n=12\right.$ in each group). ${ }^{\mathrm{a}} p<0.01$ versus the SHAM group; ${ }^{\mathrm{b}} p<0.05$ vs. the OVX group; ${ }^{c} p<0.01$ vs. the OVX group.

Figure 3. Representative sample from each group: 3D architecture of alveolar bone beneath the lowest point of the first molar crown. (a) SHAM; (b) OVX; (c) E2; (d) RDE.
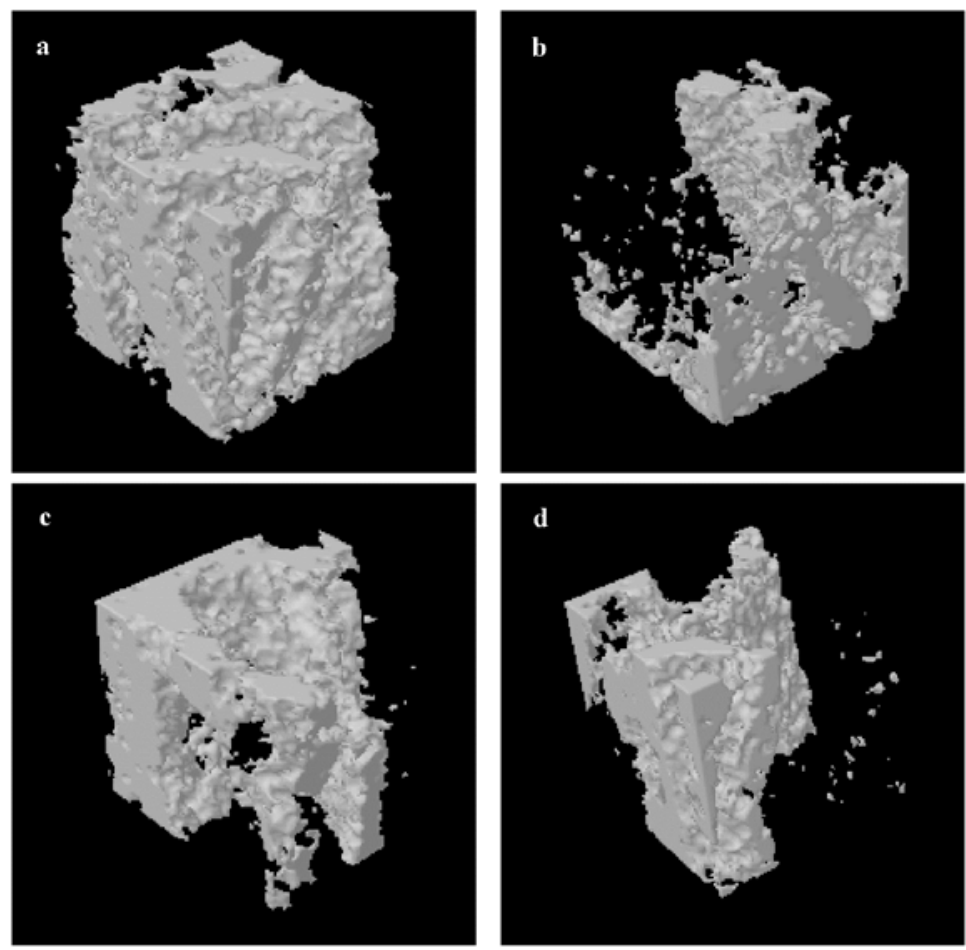


\subsection{Effect of RDE on Histological Morphology of Alveolar Bone}

We performed a histological observation of the alveolar bone region of first molar in rats. Figure 4 shows a representative histological section of the alveolar bone region of the first molar of the sham-operated or OVX rats treated with vehicle, E2 or RDE. Section from rats from with sham operation showed thick alveolar bone with relatively scant marrow space (Figure 4a). OVX markedly reduced alveolar bone volume and increased the extent of the marrow space (Figure 4b). E2 or RDE treatment for 12 weeks markedly increased alveolar bone volume (Figure 4c,d), but E2 had a more potent effect than RDE. The results from micro-CT and histological observation were reciprocal confirmation.

Figure 4. Histological observation of the alveolar bone region of the first molar in rats. The sections were stained with hematoxylin and eosin. (a) SHAM; (b) OVX; (c) E2; (d) RDE. Scale bar $=1 \mathrm{~mm}$. Original magnification, $4 \times$.
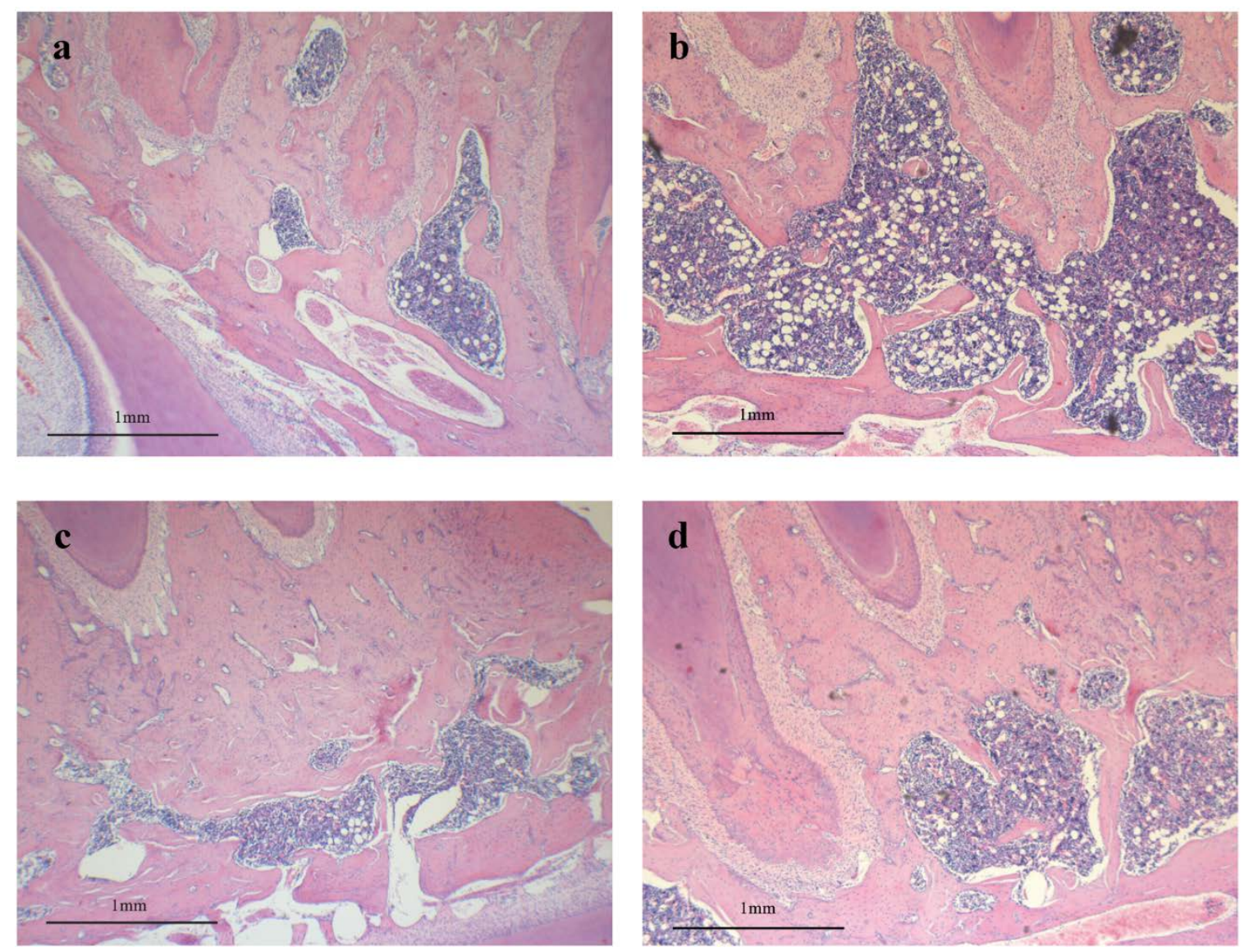

\subsection{Effect of RDE on Gene Expression Profile}

The array results demonstrated that the expression of 383 genes was altered ( $\geq 3$-fold) between the alveolar bone from the RDE and OVX group rats. Specifically, 207 genes were upregulated, and 176 genes were downregulated. The differentially expressed genes are listed in the Supplementary Materials. 


\subsection{Pathway Analysis of Differentially Expressed Genes}

We characterized differentially expressed genes into functions and signaling pathways by using IPA software according to the Ingenuity Pathways Knowledge Base (IPKB). These signaling pathways were ranked according to the IPA calculated scores, which are based on the significance of the involved genes. Based on IPKB, the signature genes were classified into pathways by IPA. Among these, the top five pathways were identified by IPA with significance values of less than 0.05 (Table 3). Of the top five pathways, two pathways ("role of osteoblasts, osteoclasts and chondrocytes in rheumatoid arthritis" and "Wnt/ $\beta$-catenin signaling") were associated with bone metabolism, and the latter pathway was included in the former. In the present study, we selected the following genes, interleukin 1 receptor antagonist (Il1rn), secreted frizzled-related protein 2 (Sfrp2), secreted frizzled-related protein 4 (Sfrp4), wingless-type MMTV integration site family, member 7A (Wnt7a), frizzled-related protein (Frzb), frizzled family receptor 2 ( Fzd2), bone morphogenetic protein 1 (Bmp1), transcription factor 3 (Tcf3), secreted phosphoprotein 1 (Spp1, known as osteopontin, Opn), mitogen-activated protein kinase 14 (Mapk14), colony-stimulating factor 1 receptor (Csf1r) and protein tyrosine kinase 2 beta ( $P t k 2 b)$, involved in the two significant signaling pathways associated with bone metabolism (Figure 5), for additional evaluation.

Table 3. Top canonical pathways associated with differentially expressed genes between the RDE group and OVX group.

\begin{tabular}{lcc}
\hline Ingenuity Canonical Pathways & $\boldsymbol{p}$-Value & Number of Molecules \\
\hline Role of osteoblasts, osteoclasts and chondrocytes in rheumatoid arthritis & $1.23 \times 10^{-3}$ & 12 \\
Role of Wnt/GSK-3 $\beta$ signaling in the pathogenesis of influenza & $1.34 \times 10^{-2}$ & 4 \\
Basal cell carcinoma signaling & $2.02 \times 10^{-2}$ & 4 \\
Human embryonic stem cell pluripotency & $2.33 \times 10^{-2}$ & 6 \\
Wnt/ $\beta$-catenin signaling & $4.07 \times 10^{-2}$ & 6 \\
\hline
\end{tabular}

\subsection{Confirmation of Differential Levels of Gene Expression by qRT-PCR}

To confirm the differential gene expression detected by the microarray analysis and pathway analysis, we sampled 12 genes (Il1rn, Sfrp2, Sfrp4, Wnt7a, Frzb, Fzd2, Bmp1, Tcf3, Spp1, Mapk14, Csf1r and $P t k 2 b$ ) involved in the significant signaling pathway "role of osteoblasts, osteoclasts and chondrocytes in rheumatoid arthritis" and "Wnt/ $\beta$-catenin signaling" for verification using qRT-PCR. Figure 6 presents comparative changes in these genes as determined by microarray and qRT-PCR. In all of these cases, the results from qRT-PCR generally agreed with the changes in the microarray analysis, although the absolute degree of change differed markedly between both methods. 
Figure 5. Schematic diagram illustrating the role of osteoblasts and osteoclasts in an anti-osteopenic effect of RDE. The downregulated genes appear in blue, and the upregulated genes appear in red. The white indicates the genes that are not specified, but that are incorporated into the network through relationships.

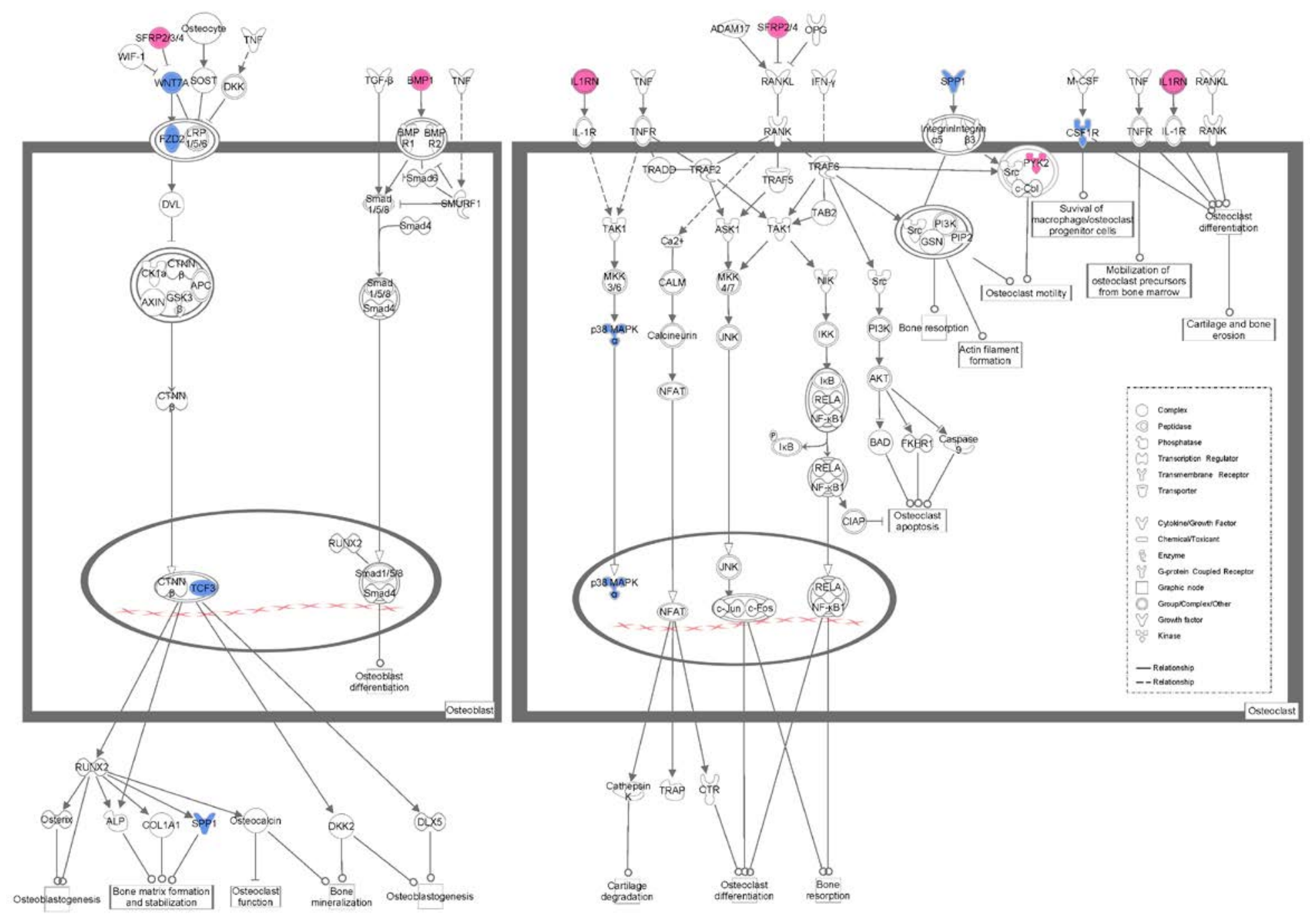


Figure 6. Validation of 12 differentially expressed genes identified by microarray and ingenuity pathway analysis (IPA) in a replicated experiment by qRT-PCR. (a)-(I) Effect of RDE on the expressions of Wnt7a, Fzd2, Tcf3, Spp1, Mapk14, Frzb, Sfrp2, Sfrp4, Il1rn, Ptk2b, Bmp1 and Csf1r, respectively. * $p<0.05 ; * * p<0.01$, compared with the OVX group.
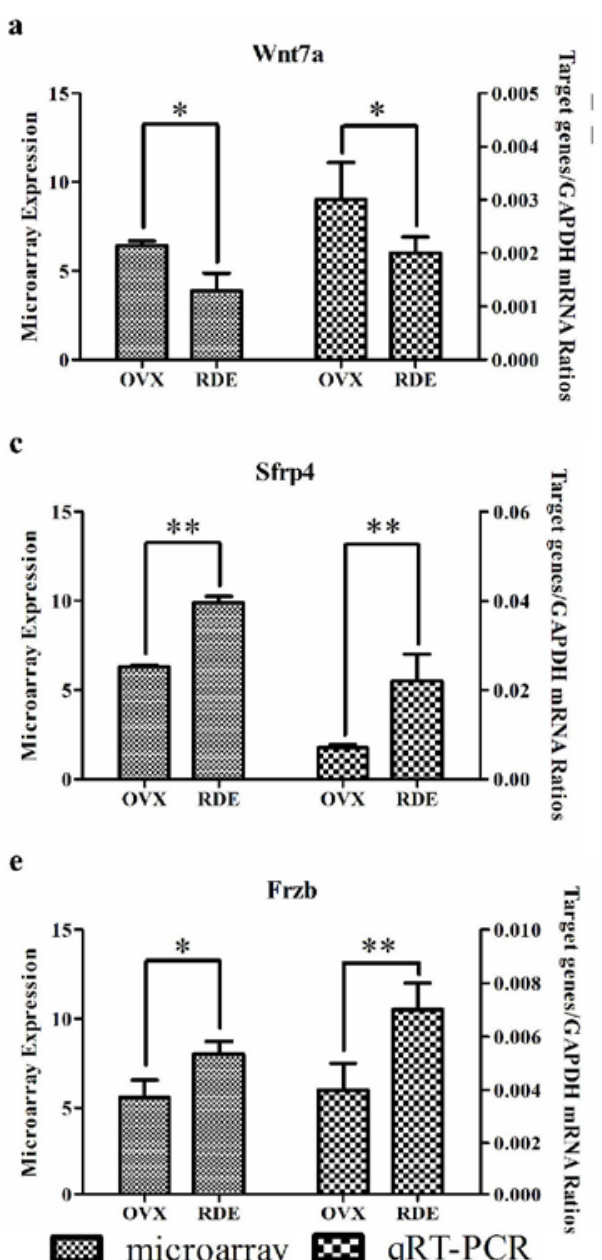

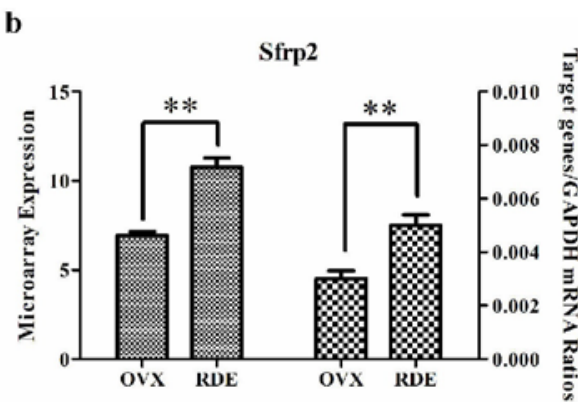

d
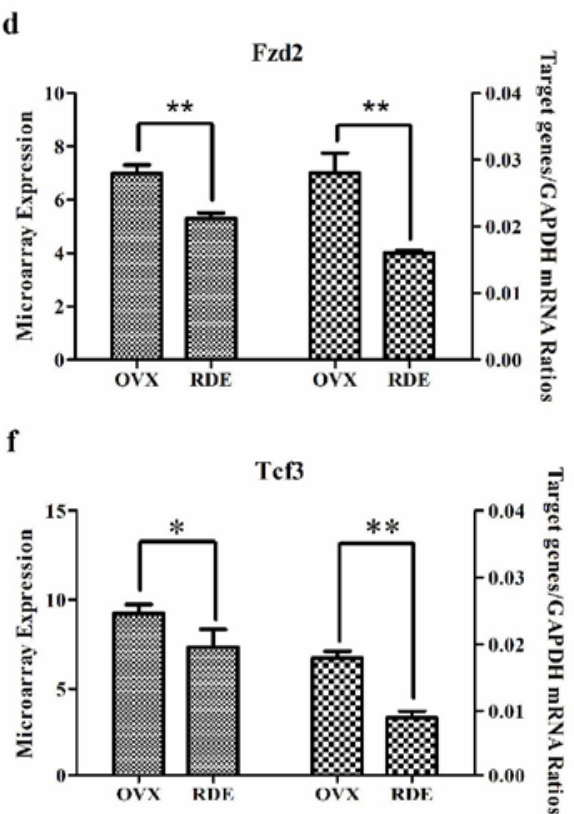

Spp1

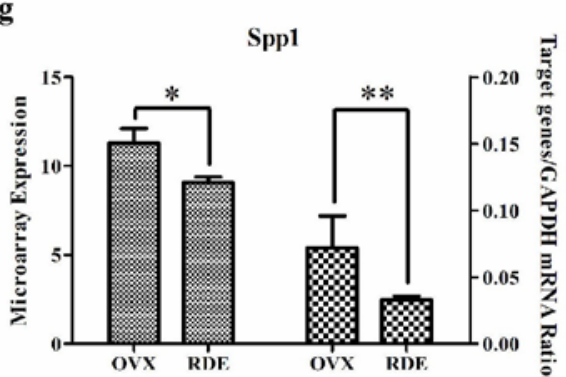

\begin{abstract}
i
\end{abstract}

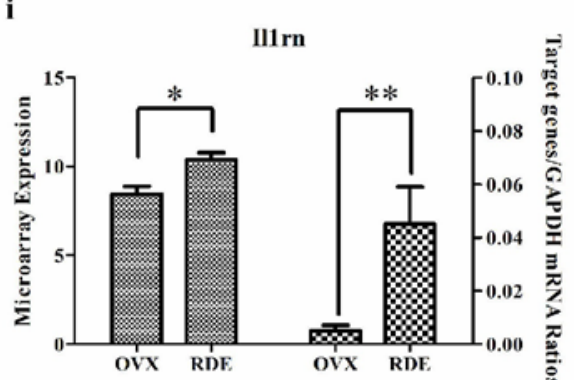

k

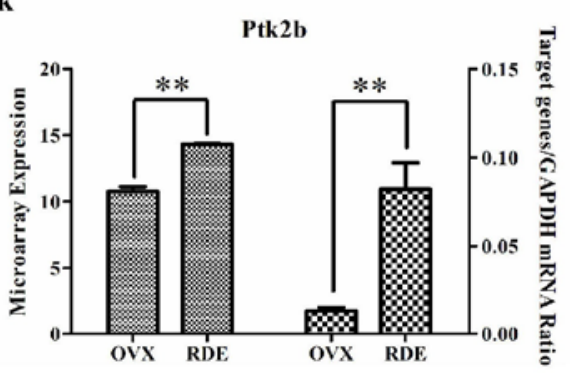

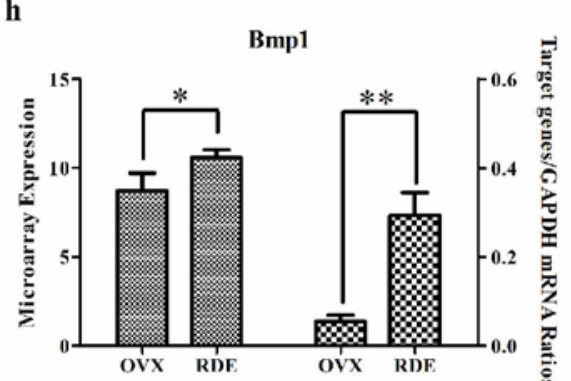

j

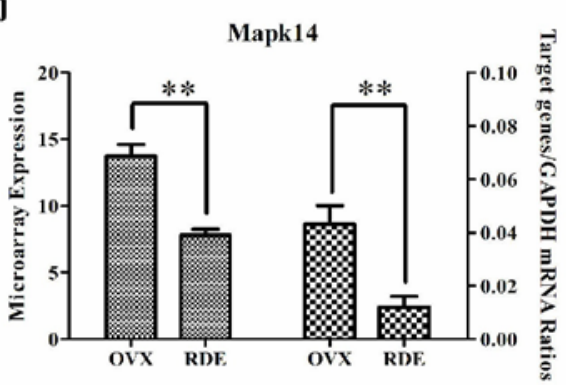

I

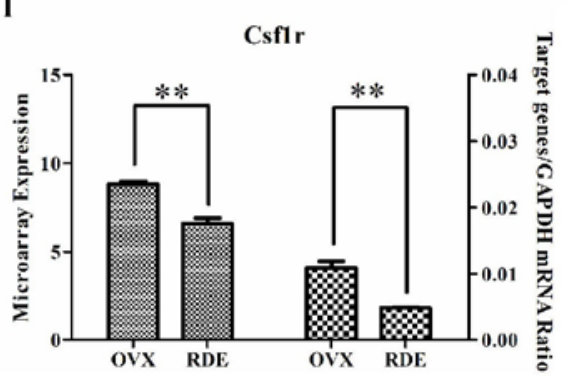




\subsection{Confirmation of Proteins by Western Blotting}

We further assessed changes in the protein expression of two genes, Tc $\beta 3$ and Mapk14. We found that changes in the protein expression of Tcf-3 and p38 $\alpha$ agreed with the mRNA expression and were downregulated in the RDE-treated rats (Figure 7).

Figure 7. Validation of two proteins by western blotting: Effect of RDE on the expressions of Tcf-3 and p38 $\alpha$. Actin was employed as the housekeeping gene. Representative blots are shown (left). Band intensities were quantified from six independent samples using densitometry (right). ${ }^{\mathrm{a}} p<0.01$ vs. the SHAM group; ${ }^{\mathrm{b}} p<0.01$ vs. the OVX group.
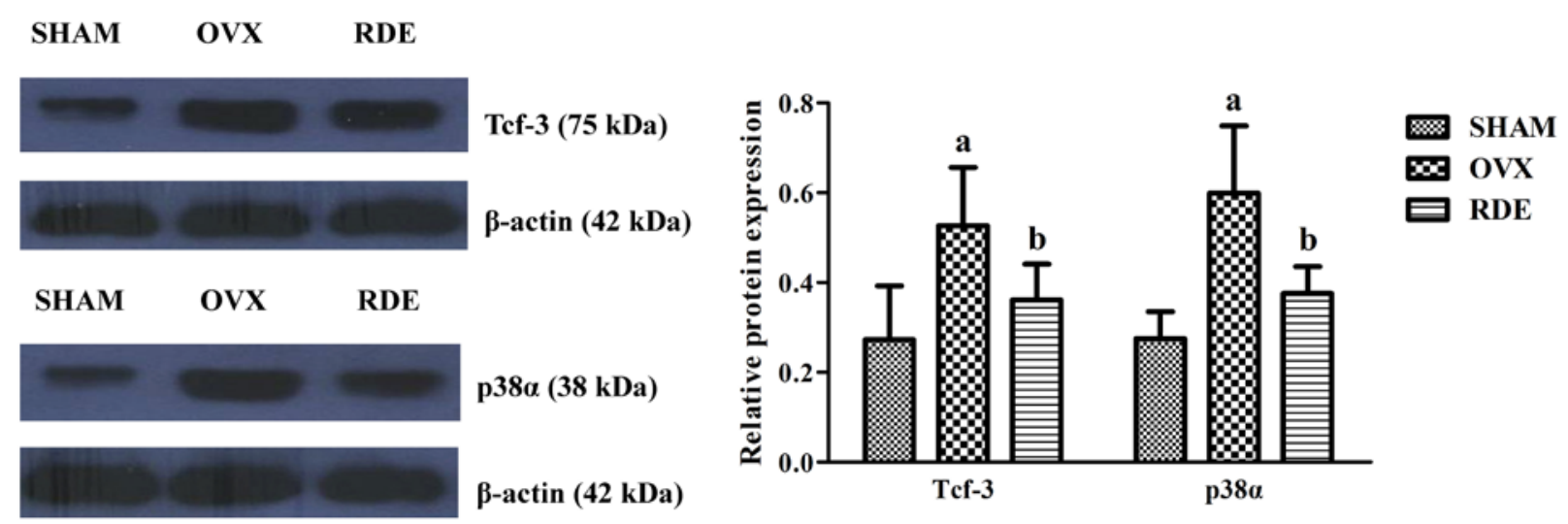

\section{Discussion}

The alveolar bone is a special tissue originating from the dental sac and constantly undergoes chronic inflammatory stimuli or mechanical stress, such as occlusal pressure. Furthermore, the alveolar bone has a very short biological metabolic cycle, and its metabolic mechanism is different from that of other bones to some extent [24]. A large cohort of postmenopausal women who show systemic bone loss and osteoporosis also carry a high risk of tooth and oral bone loss. Such tooth loss and periodontal disease may be associated with alveolar bone resorption $[25,26]$. Our previous study showed that treatment with RDE protects against bone loss in the peripheral skeleton in OVX rats [18]. However, the effect of RDE on alveolar bone remained unclear. In the present study, we evaluated the effect of RDE on alveolar bone in rats after OVX.

Following ovariectomy, BMD was markedly decreased, due to an increase in alveolar bone turnover in the OVX rats compared to the SHAM rats. In contrast, treatment with the E2 or RDE increased the BMD of the alveolar bone compared to the OVX group.

Coincident and significant increases in the plasma biomarkers for the assessment of bone resorption, CTX, and, for the assessment of bone formation, P1NP indicated that the mature OVX rat is a reliable and suitable animal model for studying high turnover bone loss, such as early postmenopausal osteoporosis. The treatment with E2 or RDE for 12 weeks was able to lower the increased bone turnover, which was reflected by the significant decreases in CTX and P1NP (Figures 1 and 2).

An analysis of 3D bone microarchitecture by micro-CT showed that the alveolar bone in RDE- or E2-treated rats had less bone loss than the OVX group, which was reflected by the significant change in BV/TV, Tb.Th, Tb.N, Tb.Sp and SMI. E2 had a more potent anti-osteopenic effect on alveolar bone than 
RDE (Table 2). The histological observation of alveolar bone (Figure 4) confirmed the results of micro-CT assessment (Figure 3).

The results from the assays of BMD, micro-CT, bone turnover biomarkers and histological observation showed that RDE had a substantial anti-osteopenic effect on alveolar bone.

To explore the mechanism of the anti-osteopenic effect of RDE on alveolar bone, we screened the differential expression of genes by microarray and found key pathways with IPA. In our study, we succeeded in identifying 383 genes, which were differentially expressed in the alveolar bone of the RDE group compared to the OVX group. Table 3 shows the top five canonical pathways from the hundreds of canonical pathways $(p<0.05)$. Of the top five pathways, two pathways were related to bone metabolism. From Figure 5, we observed that the Wnt/ $\beta$-catenin signaling pathway in osteoblasts and p38 MAPK signaling pathway in osteoclasts were downregulated after the RDE treatment.

The canonical Wnt/ $\beta$-catenin signaling pathway is involved in osteoblast proliferation and bone matrix formation, stabilization and mineralization $[27,28]$ and is initiated by the binding of Wnt ligands to the complex compromised of frizzled homolog proteins (FZDs) and low-density lipoprotein receptor-related proteins 5/6 (LRP5/6) [29]. The resultant signals prevent $\beta$-catenin phosphorylation by a multiprotein complex composed of adenomatous polyposis coli (APC), glycogen synthase kinase $3 \beta$ (GSK-3 $\beta$ ), casein kinase 1 (CK-1) and axins, causing its proteasomal degradation. The $\beta$-catenin associates with T-cell factor (TCF)/lymphocyte enhancer transcription factors (LEF) to activate target genes [30]. The secreted frizzled-related proteins (sFRP1 to sFRP5) are a family of soluble proteins that are structurally related to FZDs. Because of their homology with the Wnt-binding domain on the FZDs, SFRPs were immediately characterized as antagonists that bind to Wnt proteins to prevent signal activation [31].

In the canonical Wnt/ $\beta$-catenin signaling pathway, the TCF/LEF family transcription factors (e.g., TCF3) played a pivotal role in promoting bone-specific gene expression, such as Runt-related gene 2 (RUNX2) [32]. RUNX2 is a multifunctional transcription factor that controls skeletal development by regulating the differentiation of osteoblasts and the expression of many extracellular matrix protein genes [33]. During osteoblast differentiation, RUNX2 upregulates the expression of bone matrix protein genes, including SPP1, also known as OPN [34]. SPP1 is an early marker of periodontal tissue regeneration, which is temporally and spatially associated with intensive cell proliferation and migration in the osteogenic cell population [35]. An in vitro study showed that diosgenin could induce RUNX2regulated osteopontin protein expression in osteoblasts at low concentrations, and again, this decreased at high concentrations [36].

We found that gene expressions of Wnt7a, Fzd2, Tcf3 and Spp1 in the alveolar bone were downregulated, and Frzb, Sfrp2 and Sfrp4 were upregulated after treatment with RDE (Figure 6). This regulation of gene expression led to an inhibitory effect on the Wnt/ $\beta$-catenin signaling, which plays a pivotal role in osteoblastogenesis. Our qRT-PCR analyses of Wnt7a, Frzb, Fzd2, Tcf3, Spp1, Sfrp2 and Sfrp4 expressions (Figure 6a-g) and western blotting analysis of Tcf-3 (Figure 7) convinced us that RDE could decrease the high bone formation in the alveolar bone of OVX rats, which results from ovariectomy by attenuating the canonical Wnt/ $\beta$-catenin signaling, and that the results of the microarray and IPA were credible.

MAPK family members are classified into three groups: the ERK, JNK and p38 MAPK groups. Phosphorylation of p38 MAPK (p38 $\alpha$ coded by Mapk14, p38 $\beta$ coded by Mapk11 and p38 $\gamma$ coded by 
Mapk12) by MAPK kinase (MKK) 3/6 results in p38 MAPK activation. Activated p38 MAPK then phosphorylates transcription factor ATF2, which, in turn, induces target gene transcription $[37,38]$. p38 MAPK is primarily activated within cells involved in the inflammatory process, which, in turn, induces the synthesis of key inflammatory mediators, such as tumor necrosis factor $\alpha$ (TNF- $\alpha$ ), interleukin (IL)-1, IL-6, IL-8 and cyclooxygenase-2, either via direct gene transcription activation or messenger RNA stabilization. In the context of bone, p38 MAPK occupies a central role in the regulation of IL-1 and TNF- $\alpha$-signaling networks and NF- $\mathrm{B}$ ligand (RANKL)-induced osteoclastic bone resorption [39]. Several studies showed that p38 MAPK signaling is involved in osteoclast differentiation, which is responsible for periapical alveolar bone resorption, and that the p38 MAPK inhibitor could inhibit active alveolar bone loss in a rat periodontitis model [40,41].

We found that the expressions of Il1rn (upregulated) and Mapk14 (downregulated) in the alveolar bone were regulated after treatment with RDE (Figure 5). Our qRT-PCR analyses of Il1rn and Mapk14 expressions (Figure 6i,j) and western blotting analysis of p38 $\alpha$ (Figure 7) convinced us that RDE could decrease high bone resorption in the alveolar bone of OVX rats, which results from ovariectomy by attenuating p38 MAPK signaling, and that the results of the microarray and IPA were credible.

After the treatment of RDE, the expressions of Wnt7a, Fzd2, Tcf3, Spp1 and Mapk14 in the alveolar bone were down regulated, while the expressions of Frzb, Sfrp2, Sfrp4 and Il1rn were up regulated. On the one hand, the regulation of Wnt7a, Frzb, Fzd2, Tcf3, Spp1, Sfrp2 and Sfrp4 could inhibit osteoblastogenesis via attenuating Wnt/ $\beta$-catenin signaling. On the other hand, the regulation of Il1rn and Mapk14 could inhibit osteoclastogenesis via reducing p38 MAPK signaling. Our qRT-PCR or western blotting analyses of these gene or protein expressions convinced us that RDE could decrease the excessively high bone formation and the bone resorption synchronously in the OVX rat alveolar bone to a certain extent. This inhibitory effect of RDE was associated with the $\mathrm{Wnt} / \beta$-catenin signaling and p38 MAPK signaling. Furthermore, because rats in the RDE group had higher bone mass than those in the OVX group, we inferred that RDE had a more potent inhibitory effect on bone resorption than bone formation.

In this research, we only explored the anti-osteopenic effect of RDE in an animal model. A line of studies had to be performed before RDE became a promising alternative preventive and therapeutic agent for relieving alveolar bone loss of postmenopausal women.

\section{Conclusions}

RDE may inhibit ovariectomy-induced alveolar bone loss in rats. The mechanism for the anti-osteopenic effect of RDE may lie in the simultaneous inhibition of both bone formation and bone resorption that occurs following regulation of $\mathrm{Wnt} / \beta$-catenin signaling and p38 MAPK signaling. Our study provides evidence that the aqueous extract of Rhizoma Dioscoreae may have potential use as an oral drug for treating alveolar bone loss in postmenopausal females.

\section{Acknowledgments}

This study was supported by the National Natural Science Foundation of China (81102680, 81473450) and the Fundamental Research Funds for the Central Public Welfare Research Institutes (YZ-1409). 


\section{Author Contributions}

Study conception and design: Dahong Ju, Gary Guishan Xiao and Zhiguo Zhang. Preparation of aqueous extract: Dong Bai and Wenlai Wang. Acquisition of data: Zhiguo Zhang, Yan Li, Jinghua Pan, Hong Liu, Shaojun Wang and Lihua Xiang. Analysis and interpretation of data: Zhiguo Zhang, Dong Bai and Wenlai Wang. Drafting of manuscript: Dahong Ju, Gary Guishan Xiao and Zhiguo Zhang.

\section{Conflicts of Interest}

The authors declare no conflict of interest.

\section{References}

1. Zhang, Z.; Song, C.; Fu, X.; Liu, M.; Li, Y.; Pan, J.; Liu, H.; Wang, S.; Xiang, L.; Xiao, G.G.; et al. High-dose diosgenin reduces bone loss in ovariectomized rats via attenuation of the RANKL/OPG ratio. Int. J. Mol. Sci. 2014, 15, 17130-17147.

2. Pinkerton, J.V.; Thomas, S.; Dalkin, A.C. Osteoporosis treatment and prevention for postmenopausal women: Current and future therapeutic options. Clin. Obstet. Gynecol. 2013, 56, 711-721.

3. Lee, B.D.; White, S.C. Age and trabecular features of alveolar bone associated with osteoporosis. Oral Surg. Oral Med. Oral Pathol. Oral Radiol. Endod. 2005, 100, 92-98.

4. Streckfus, C.F.; Johnson, R.B.; Nick, T.; Tsao, A.; Tucci, M. Comparison of alveolar bone loss, alveolar bone density and second metacarpal bone density, salivary and gingival crevicular fluid interleukin-6 concentrations in healthy premenopausal and postmenopausal women on estrogen therapy. J. Gerontol. Ser. A Biol. Sci. Med. Sci. 1997, 52, M343-M351.

5. Sultan, N.; Rao, J. Association between periodontal disease and bone mineral density in postmenopausal women: A cross sectional study. Med. Oral Patol. Oral Cir. Bucal 2011, 16, e440-e447.

6. Tezal, M.; Wactawski-Wende, J.; Grossi, S.G.; Ho, A.W.; Dunford, R.; Genco, R.J. The relationship between bone mineral density and periodontitis in postmenopausal women. J. Periodontol. 2000, 71, 1492-1498.

7. Gomes-Filho, I.S.; Passos Jde, S.; Cruz, S.S.; Vianna, M.I.; Cerqueira Ede, M.; Oliveira, D.C.; dos Santos, C.A.; Coelho, J.M.; Sampaio, F.P.; Freitas, C.O.; et al. The association between postmenopausal osteoporosis and periodontal disease. J. Periodontol. 2007, 78, 1731-1740.

8. Tezal, M.; Wactawski-Wende, J.; Grossi, S.G.; Dmochowski, J.; Genco, R.J. Periodontal disease and the incidence of tooth loss in postmenopausal women. J. Periodontol. 2005, 76, 1123-1128.

9. Civitelli, R.; Pilgram, T.K.; Dotson, M.; Muckerman, J.; Lewandowski, N.; Armamento-Villareal, R.; Yokoyama-Crothers, N.; Kardaris, E.E.; Hauser, J.; Cohen, S.; et al. Alveolar and postcranial bone density in postmenopausal women receiving hormone/estrogen replacement therapy: A randomized, double-blind, placebo-controlled trial. Arch. Intern. Med. 2002, 162, 1409-1415.

10. Palomo, L.; Bissada, N.F.; Liu, J. Periodontal assessment of postmenopausal women receiving risedronate. Menopause 2005, 12, 685-690. 
11. Liu, J.; Cao, Z.; Li, C. Intermittent PTH administration: A novel therapy method for periodontitis-associated alveolar bone loss. Med. Hypotheses 2009, 72, 294-296.

12. Strom, B.L.; Schinnar, R.; Weber, A.L.; Bunin, G.; Berlin, J.A.; Baumgarten, M.; DeMichele, A.; Rubin, S.C.; Berlin, M.; Troxel, A.B.; et al. Case-control study of postmenopausal hormone replacement therapy and endometrial cancer. Am. J. Epidemiol. 2006, 164, 775-786.

13. Rossing, M.A.; Cushing-Haugen, K.L.; Wicklund, K.G.; Doherty, J.A.; Weiss, N.S. Menopausal hormone therapy and risk of epithelial ovarian cancer. Cancer Epidemiol. Biomarkers Prev. 2007, 16, 2548-2556.

14. Woo, S.B.; Hellstein, J.W.; Kalmar, J.R. Narrative corrected review: Bisphosphonates and osteonecrosis of the jaws. Ann. Intern. Med. 2006, 144, 753-761.

15. Rizzoli, R.; Reginster, J.Y.; Boonen, S.; Breart, G.; Diez-Perez, A.; Felsenberg, D.; Kaufman, J.M.; Kanis, J.A.; Cooper, C. Adverse reactions and drug-drug interactions in the management of women with postmenopausal osteoporosis. Calcif. Tissue Int. 2011, 89, 91-104.

16. Clemett, D.; Spencer, C.M. Raloxifene: A review of its use in postmenopausal osteoporosis. Drugs 2000, 60, 379-411.

17. Jiang, M.; Zhang, C.; Cao, H.; Chan, K.; Lu, A. The role of chinese medicine in the treatment of chronic diseases in China. Planta Med. 2011, 77, 873-881.

18. Zhang, Z.; Xiang, L.; Bai, D.; Fu, X.; Wang, W.; Li, Y.; Liu, H.; Pan, J.; Xiao, G.G.; Ju, D. Treatment with Rhizoma Dioscoreae extract has protective effect on osteopenia in ovariectomized rats. Sci. World J. 2014, doi:10.1155/2014/645975.

19. Li, C.M.; Dong, X.L.; Fan, X.D.; Wu, J.H.; Wang, Q.H.; Tian, X.L.; Guo, D.J.; Wong, M.S.; Qiu, T.Q.; Chan, S.W. Aqueous extract of danshen (Salvia miltiorrhiza Bunge) protects ovariectomized rats fed with high-fat diet from endothelial dysfunction. Menopause 2013, 20, 100-109.

20. Maimoun, L.; Brennan-Speranza, T.C.; Rizzoli, R.; Ammann, P. Effects of ovariectomy on the changes in microarchitecture and material level properties in response to hind leg disuse in female rats. Bone 2012, 51, 586-591.

21. Lane, N.E.; Yao, W.; Kinney, J.H.; Modin, G.; Balooch, M.; Wronski, T.J. Both hPTH(1-34) and bFGF increase trabecular bone mass in osteopenic rats but they have different effects on trabecular bone architecture. J. Bone Miner. Res. 2003, 18, 2105-2115.

22. Yang, J.; Pham, S.M.; Crabbe, D.L. High-resolution Micro-CT evaluation of mid- to long-term effects of estrogen deficiency on rat trabecular bone. Acad. Radiol. 2003, 10, 1153-1158.

23. Bouxsein, M.L.; Boyd, S.K.; Christiansen, B.A.; Guldberg, R.E.; Jepsen, K.J.; Muller, R. Guidelines for assessment of bone microstructure in rodents using micro-computed tomography. J. Bone Miner. Res. 2010, 25, 1468-1486.

24. Ikeo, T.; Goda, S.; Domae, E. Metabolism of alveolar bone. Clin. Calcium 2006, 16, 117-121.

25. Taguchi, A.; Tanimoto, K.; Suei, Y.; Wada, T. Tooth loss and mandibular osteopenia. Oral Surg. Oral Med. Oral Pathol. Oral Radiol. Endod. 1995, 79, 127-132.

26. Krall, E.A.; Garcia, R.I.; Dawson-Hughes, B. Increased risk of tooth loss is related to bone loss at the whole body, hip, and spine. Calcif. Tissue Int. 1996, 59, 433-437.

27. Li, X.; Liu, P.; Liu, W.; Maye, P.; Zhang, J.; Zhang, Y.; Hurley, M.; Guo, C.; Boskey, A.; Sun, L.; et al. Dkk2 has a role in terminal osteoblast differentiation and mineralized matrix formation. Nat. Genet. 2005, 37, 945-952. 
28. Kubota, T.; Michigami, T.; Ozono, K. Wnt signaling in bone metabolism. J. Bone Miner. Metab. 2009, 27, 265-271.

29. Nusse, R. Wnt signaling. Cold Spring Harb. Perspect. Biol. 2012, doi:10.1101/cshperspect.a011163.

30. Ueno, K.; Hirata, H.; Hinoda, Y.; Dahiya, R. Frizzled homolog proteins, microRNAs and Wnt signaling in cancer. Int. J. Cancer 2013, 132, 1731-1740.

31. Bovolenta, P.; Esteve, P.; Ruiz, J.M.; Cisneros, E.; Lopez-Rios, J. Beyond Wnt inhibition: New functions of secreted frizzled-related proteins in development and disease. J. Cell Sci. 2008, 121, 737-746.

32. Niehrs, C. The complex world of Wnt receptor signalling. Nat. Rev. Mol. Cell Biol. 2012, 13, 767-779.

33. Komori, T. Regulation of bone development and extracellular matrix protein genes by RUNX2. Cell Tissue Res. 2010, 339, 189-195.

34. Zhang, Z.; Deepak, V.; Meng, L.; Wang, L.; Li, Y.; Jiang, Q.; Zeng, X.; Liu, W. Analysis of HDAC1-mediated regulation of RUNX2-induced osteopontin gene expression in C3h10t1/2 cells. Biotechnol. Lett. 2012, 34, 197-203.

35. Lekic, P.; Sodek, J.; McCulloch, C.A. Relationship of cellular proliferation to expression of osteopontin and bone sialoprotein in regenerating rat periodontium. Cell Tissue Res. 1996, 285, 491-500.

36. Alcantara, E.H.; Shin, M.Y.; Sohn, H.Y.; Park, Y.M.; Kim, T.; Lim, J.H.; Jeong, H.J.; Kwon, S.T.; Kwun, I.S. Diosgenin stimulates osteogenic activity by increasing bone matrix protein synthesis and bone-specific transcription factor RUNX2 in osteoblastic MC3T3-E1 cells. J. Nutr. Biochem. 2011, 22, 1055-1063.

37. Cobb, M.H.; Goldsmith, E.J. How map kinases are regulated. J. Biol. Chem. 1995, 270, 14843-14846.

38. Whitmarsh, A.J.; Davis, R.J. Transcription factor AP-1 regulation by mitogen-activated protein kinase signal transduction pathways. J. Mol. Med. Berl. 1996, 74, 589-607.

39. Wei, S.; Kitaura, H.; Zhou, P.; Ross, F.P.; Teitelbaum, S.L. IL-1 mediates TNF-induced osteoclastogenesis. J. Clin. Investig. 2005, 115, 282-290.

40. Rogers, J.E.; Li, F.; Coatney, D.D.; Otremba, J.; Kriegl, J.M.; Protter, T.A.; Higgins, L.S.; Medicherla, S.; Kirkwood, K.L. A p38 mitogen-activated protein kinase inhibitor arrests active alveolar bone loss in a rat periodontitis model. J. Periodontol. 2007, 78, 1992-1998.

41. Zhang, R.; Wang, L.; Peng, B. Activation of p38 mitogen-activated protein kinase in rat periapical lesions. J. Endod. 2008, 34, 1207-1210.

(C) 2014 by the authors; licensee MDPI, Basel, Switzerland. This article is an open access article distributed under the terms and conditions of the Creative Commons Attribution license (http://creativecommons.org/licenses/by/4.0/). 